\title{
Sliding Mode Control Design for a Class of SISO Systems with Uncertain Sliding Surface
}

\author{
Guofeng Wang, Kai Zheng, Xingcheng Wang, and Shuanghe Yu \\ Information Science and Technology College, Dalian Maritime University, Dalian 116026, China \\ Correspondence should be addressed to Kai Zheng; kdmu@ieee.org
}

Received 1 May 2013; Accepted 2 June 2013

Academic Editor: Lijun Zhang

Copyright (C) 2013 Guofeng Wang et al. This is an open access article distributed under the Creative Commons Attribution License, which permits unrestricted use, distribution, and reproduction in any medium, provided the original work is properly cited.

\begin{abstract}
The problem of designing a sliding mode controller with uncertain sliding surface for a class of uncertain single-input-singleoutput systems is studied. The design case is handled by using the invariant transformation first in order to separate the sliding mode and the reaching mode of the sliding mode control system. It is shown that the sliding mode design needs not to consider the uncertainties of the sliding surface, which can be handled in the reaching phase design. The results generalize the robust design of the reaching phase such that one specific reaching phase design may agree with several sliding surfaces.
\end{abstract}

\section{Introduction}

Sliding mode control (SMC) is one of the well-known powerful design tools for the problem of the deterministic control of uncertain systems, since it has a significant insensitive feature to handle internal parameter variations and external disturbances [1-4]. The typical SMC design consists of two stages: sliding phase design and reaching phase design. Usually, a hyperplane, namely, sliding surface, is first designed to provide the desired behavior for the closed-loop system during sliding mode. The sliding surface is also considered to be the switching condition during the reaching phase design that employs a discontinuous control law to force all the trajectories to reach the sliding surface and remain on it. As long as the sliding mode is realized, the system achieves invariant properties with respect to parameter perturbations and external matched disturbance. These design issues have received the most attention in the literature, such as [3-7], both in the fields of theory and practice.

The sliding phase design obtains extensively attention, since it not only determines the desired performance but also actually provides the design objective of the second stage [2, 3]. Many researches are concerned with the switching surface design problem in the view of the system design objectives, such as stability [8-10], performance index minimization [2], chattering reduction [11], and robustness [5]. It is observed that all the aforementioned works concentrate on the switching surfaces that are predetermined. In other words, the user-chosen sliding surface defines an invariant manifold of the closed-loop system so that the design performance can be realized. However, during the realization of the sliding surface, the uncertainties may occur, if the sliding surface relates to the uncertain physical quantities. Such uncertainties may have an influence on the performance of the closed-loop system and even cause failure of the reaching phase design. This robust design situation of SMC is always ignored in the published papers. Therefore, it is necessary to reconsider the robustness of two design stages of SMC in the presence of the uncertainties of the sliding surface.

In fact, recent results of nonsmooth control show that the sliding surface can be designed more flexibly. The authors in [12] developed a class of sliding surfaces generated by combining several linear hypersurfaces. The paper shows the fact that one reaching phase design may agree with several linear sliding surfaces, as well as their combinations. Moreover, the authors in [13] proposed a set of new SMC design rules, called cone design conditions. Under the cone conditions, the linear Lipschitz continuous hypersurface, whose gradient varies along the sliding regions, can be used as the sliding surface so that the dynamics of the sliding motion 
can be regulated according to the regions of the surface. It also implies that the reaching phase can be designed robust to the sliding surface variation. These results actually indicate the robustness of the reaching phase. Therefore, the design case of the SMC with an uncertain sliding surface can be considered as the robust design case of the reaching law.

Robust design problem is reconsidered in this paper to handle the design case of SMC with an uncertain linear sliding surface for a class of uncertain single-input-singleoutput (SISO) systems. An invariant transformation is first introduced to separate the sliding mode and the reaching mode such that it is not necessary to consider the sliding surface and its uncertainties when checking the stability of the sliding mode. Thus, the sliding mode can be designed without consideration of the uncertain sliding surface. The robust design for the uncertainties of the sliding surface is presented in the second design stage, since the uncertainties of the sliding surface are transferred to the reaching phase. The design procedure proposed in this paper is different from the usual SMC design, since the sliding mode design is carried out without an explicit sliding surface. It means that the sliding mode controller obtained by this method also agrees with several sliding surfaces. This advantage will be illustrated and verified by numerical simulations.

The rest of the paper is organized as follows. Section 2 formulates the robust design problem of SMC with an uncertain sliding surface. Section 3 introduces the two stages of the robust SMC design. In Section 4, numerical simulations are carried out to show the advantages of our design. Finally, some conclusions are made in Section 5.

Notations. The following notations are used in this paper. $I_{n}$ is the $n$ by $n$ identity matrix. $E_{n}$ denotes the $n \times 1$ vector of $n$ ones, that is, $E_{n}=\left[\begin{array}{llll}1 & 1 & \cdots & 1\end{array}\right]^{T}$, while $O_{n \times m}$ denotes the $n \times m$ matrix of zeros. Set $\operatorname{sgn}(x)=1$ for $x \geq 0$ and -1 , otherwise. The Euclidean norm of a vector $x$ and the corresponding induced normal of a matrix $A$ are denoted by $\|x\|$ and $\|A\|$, respectively. Moreover, the definition for the solution of differential equations in this paper agrees with Filippov's definition [14].

\section{Problem Formulation}

Robust design problem is formulated in this section in order to handle the uncertain sliding surface of SMC. Before the design problem is proposed, a simple example of DC-DC buck converter is presented to show that there actually exist the uncertainties of the sliding surface.

2.1. Uncertain Sliding Surface: A Simple Example. DC-DC buck converter is a wildly used power electronic device. Figure 1 shows the typical circuit of the converter $[7,15]$, where $i_{l}$ and $v_{c}$ are the inductor current and the capacitor voltage, respectively; $V_{\text {in }}$ is the input voltage source; $R_{c}, R_{l}$, and $R_{l d}$ denote the resistance of the capacitor $C_{c}$, the inductor $L_{l}$, and the uncertain load, respectively. The switch $S$ is considered as the ideal switch.

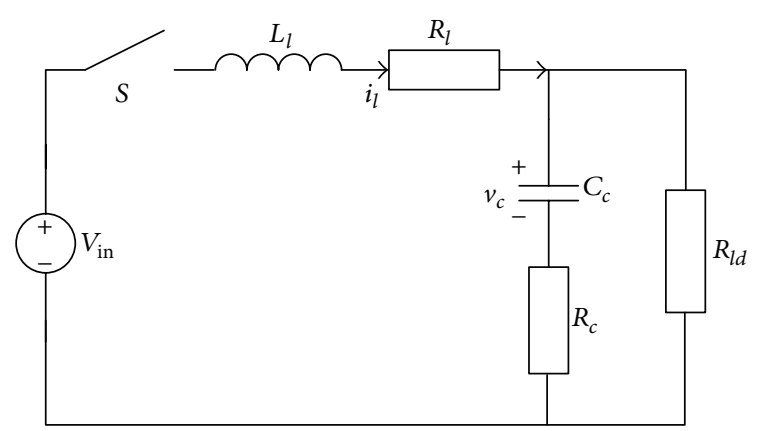

FIGURE 1: DC-DC buck converter.

The buck converter can be described by the following dynamic equation:

$$
\begin{aligned}
& {\left[\begin{array}{c}
\dot{x}_{1} \\
\dot{x}_{2}
\end{array}\right]=} {\left[\begin{array}{cc}
-\frac{1}{L_{l}}\left(R_{l}+\frac{R_{l d} R_{c}}{R_{l d}+R_{c}}\right) & -\frac{R_{l d}}{L_{l}\left(R_{l d}+R_{c}\right)} \\
\frac{R_{l d}}{C_{c}\left(R_{l d}+R_{c}\right)} & -\frac{1}{C_{c}\left(R_{l d}+R_{c}\right)}
\end{array}\right]\left[\begin{array}{l}
x_{1} \\
x_{2}
\end{array}\right] } \\
&+\left[\begin{array}{c}
\frac{1}{L} \\
0
\end{array}\right] \frac{V_{\text {in }}}{2}[1+\operatorname{sgn}(S)],
\end{aligned}
$$

where $X=\left[\begin{array}{ll}x_{1} & x_{2}\end{array}\right]^{T}=\left[\begin{array}{ll}i_{l} & v_{c}\end{array}\right]^{T}$. The output is the load voltage that can be expressed by

$$
y=C X,
$$

where $C=\left[\begin{array}{ll}c_{1} & c_{2}\end{array}\right]=\left[R_{l d} R_{c} /\left(R_{l d}+R_{c}\right) R_{l d} /\left(R_{l d}+R_{c}\right)\right]$. In order to obtain the desired output of the converter, the switching logic is applied to the switch. One of the simplest switching logics is generated from the voltage error; that is,

$$
S=y_{d}-y=y_{d}-C X .
$$

If the sliding mode occurs, that is, $S=0$, the system can be simplified as

$$
\begin{aligned}
& \dot{x}_{2}=-\frac{c_{2}}{c_{1} C_{c}} x_{2}+\frac{c_{2}}{c_{1} C_{c}} y_{d}, \\
& x_{1}=-\frac{c_{2}}{c_{1}} x_{2}+\frac{1}{c_{1}} y_{d} .
\end{aligned}
$$

Then, the sliding mode is asymptotically stable so that the desired load voltage can be realized. However, due to the uncertain switching surface whose parameters, $c_{1}$ and $c_{2}$, are related the physical quantities $R_{c}, R_{l}$, and $R_{l d}$, the dynamics of the sliding mode is also uncertain.

Moreover, the uncertain switching surface also causes the region of the sliding mode to vary. Consider the existence condition of the sliding mode proposed by Filippov [14]. The projection of the vector field on the normal of the switching surface satisfies

$$
\Omega: \begin{cases}x_{1}<k x_{2}+b, & \text { for } S>0, \\ x_{1}>k x_{2}, & \text { for } S<0,\end{cases}
$$


where $k=-c_{2} a_{21} / c_{1} a_{11}, b=V_{\text {in }} / a_{11} L$, and $a_{11}$ and $a_{21}$ are corresponding components of the system characteristic matrix of (1). It follows that the region $\Omega$ boundaries are uncertain.

This example shows that if there are uncertainties in the switching surface of SMC, the following consequences may happen:

(1) the dynamics of the sliding mode may be uncertain,

(2) the region of the sliding mode existence may be uncertain.

For a realistic SMC system design, the first consequence may cause an unstable sliding mode, and the second may cause failure of the reaching phase design. In the following, we consider the SMC design problem with a class of uncertain switching surfaces.

2.2. SMC Design Problem with an Uncertain Switching Surface. The plant considered in this paper is a class of uncertain SISO systems, which can be represented as follows:

$$
\dot{X}=F(X, t, u)=A_{0} X+\Delta A(X, t) X+B_{0} u+\Delta B(X, t) u,
$$

where $X \in \mathbb{R}^{n}$ expresses the state, $u \in \mathbb{R}$ is the control input, and $\Delta A(X, t)$ and $\Delta B(X, t)$ represent the uncertainty of the linear portion and the input matrix uncertainty, respectively. $A \in \mathbb{R}^{n \times n}$ is the system characteristic matrix, and $B \in \mathbb{R}^{n}$ is the input vector. The uncertain sliding surface is a linear hypersurface and can be defined as follows:

$$
S \triangleq\{X: \sigma(X)=\Xi X=0\},
$$

where $\Xi$ is a $1 \times n$ time-invariant vector with uncertain components.

We will assume the following to be valid:

(A1) the pair $\left(A_{0}, B_{0}\right)$ is stabilizable,

(A2) the state $X$ and the value of $\sigma(X)$ are available,

(A3) for existence purposes, $\Delta A(X, t)$ and $\Delta B(X, t)$ are continuous on $X$ and piecewise continuous on $t$,

(A4) $\triangle A(X, t)$ is of the form $N \widetilde{A}(X, t) M$ where $\widetilde{A}(X, t)$ is unknown but bounded as $\|\widetilde{A}(X, t)\| \leq 1$ for all $(X, t) \in \mathbb{R}^{n} \times \mathbb{R}$, and $N$ and $M$ are known matrices of appropriate dimensions,

(A5) there exist, function $\widetilde{B}(\cdot): \mathbb{R}^{n} \times \mathbb{R} \rightarrow \mathbb{R}$ such that, for all $(X, t) \in \mathbb{R}^{n} \times \mathbb{R}$,

$$
\Delta B(X, t)=B_{0} \widetilde{B}(X, t)
$$

and $|\widetilde{B}(X, t)| \leq \epsilon$, where $\epsilon$ is a known constant,

(A6) the uncertain vector $\Xi$ can be rewritten in the form of

$$
\Xi=E_{n}^{T}\left(I_{n}+\widetilde{\Xi}\right) \Xi_{0},
$$

where $\Xi_{0}$ and $\widetilde{\Xi}$ are two $n \times n$ matrices of the certain and the uncertain parts of the switching surface, respectively. There exists a positive number $\rho$ such that $\|\widetilde{\Xi}\|<\rho$. Moreover, $E_{n}^{T} \Xi_{0} B_{0} \neq 0$, which implies $\Xi B_{0} \neq 0$,

(A7) the boundaries of the uncertainties in the input and the sliding surface satisfy

$$
q=\rho+\epsilon \rho+\epsilon<1 .
$$

With the above structural assumption, the model uncertainties can be lumped and the system can be rewritten as

$$
\dot{X}=F(X, t, u)=A_{0} X+N \widetilde{A} M X+B_{0}(1+\widetilde{B}) u .
$$

For the system (11), two design objectives as follows need to be achieved for the sliding mode controller with the uncertain sliding surface (7).

(P1) Find the stable sliding mode that is invariant to the uncertain sliding surface (7).

(P2) Construct a discontinuous reaching law that ensures the trajectories approaching to the sliding surface in finite time.

Obviously, (P1) and (P2) relate to the two stages of the typical SMC design such that a sliding mode controller can be obtained if (P1) and (P2) are achieved. In the following section, a new SMC design methodology is introduced to develop the required controller.

\section{SMC Design with Uncertain Switching Surface}

According to the previous analysis, one can find out that the two design stages of the traditional SMC are coupled with the predetermined sliding surface. This section presents a method using invariant transformation to decouple the connection between the two design stages so that the design objectives can be achieved.

3.1. Sliding Phase Design. In order to obtain an asymptotically stable sliding mode, it is necessary to deduce the dynamics of the system on the sliding surface. Actually, since the sliding dynamics is not a new system but the special case of the original system on the constraint, it can be detached from the original system through the invariant transformation. Define a transformation as follows:

$$
Z=\left[\begin{array}{c}
z_{1} \\
\sigma
\end{array}\right]=\Gamma X=\left[\begin{array}{c}
H^{T} \\
\Xi
\end{array}\right] X,
$$

where $z_{1} \in \mathbb{R}^{n-1}, \Gamma$ is an invertible transformation matrix, and the matrix $H^{T} \in \mathbb{R}^{(n-1) \times n}$ is an orthogonal complement of $B_{0}$ and will be determined in the following. With such an invariant transformation, the system (11) can be transformed into

$$
\left[\begin{array}{c}
\dot{z}_{1} \\
\dot{\sigma}
\end{array}\right]=\Gamma\left(A_{0}+N \widetilde{A} M\right) \Gamma^{-1}\left[\begin{array}{c}
z_{1} \\
\sigma
\end{array}\right]+\left[\begin{array}{c}
0 \\
\Xi B_{0}
\end{array}\right](1+\widetilde{B}) u .
$$


Let $\bar{H}$ be an $n \times(n-1)$ matrix that satisfies $H^{T} \bar{H}=I_{n-1}$ and $\Xi_{0} \bar{H}=O_{n \times(n-1)}$. Then $\Gamma^{-1}$ can be selected as $\left[\begin{array}{ll}\bar{H} & B_{0}\left(\Xi B_{0}\right)^{-1}\end{array}\right]$. Thus, the above equation can be rewritten as follows:

$$
\begin{aligned}
& {\left[\begin{array}{c}
\dot{z}_{1} \\
\dot{\sigma}
\end{array}\right]} \\
& =\left[\begin{array}{cc}
H^{T}\left(A_{0}+N \widetilde{A} M\right) \bar{H} & H^{T}(A+N \widetilde{A} M) B_{0}\left(\Xi B_{0}\right)^{-1} \\
\Xi\left(A_{0}+N \widetilde{A} M\right) \bar{H} & \Xi(A+N \widetilde{A} M) B_{0}\left(\Xi B_{0}\right)^{-1}
\end{array}\right] \\
& \quad \times\left[\begin{array}{c}
z_{1} \\
\sigma
\end{array}\right]+\left[\begin{array}{c}
0 \\
\Xi B_{0}
\end{array}\right](1+\widetilde{B}) u .
\end{aligned}
$$

From the system (14), which is actually the system (11) in the new coordinates $z_{1}$ and $\sigma$, it can be found that the system will be governed by the dynamics of $z_{1}$, if the sliding mode occurs, that is, $\dot{\sigma}=\sigma=0$, that is,

$$
\dot{z}_{1}=H^{T}\left(A_{0}+N \widetilde{A} M\right) \bar{H} z_{1} .
$$

The differential equation (15) expresses an uncertain autonomous system. It is apparent that the system (15) is independent of the selection of the sliding surface. It can be summarized as the following lemma.

Lemma 1. The sliding mode of the sliding mode control system (11) can be expressed by (15) with the invariant transformation (12), and the sliding mode is independent of the sliding surface (7).

Therefore, the stability of the sliding mode is only related to the matrices $H$ and $\bar{H}$. According to the results of [16], the system (15) is stable if and only if there exists a positivedefinite matrix $P$ such that

$$
H^{T} A_{0} \bar{H} P+P \bar{H}^{T} A_{0}^{T} H+H^{T} N N^{T} H+P \bar{H}^{T} M^{T} M \bar{H} P<0 .
$$

This condition can be translated into a linear matrix inequality (LMI). If $P$ is chosen in the form of $P=H^{T} K H$, then $\bar{H}$ can be calculated as $\bar{H}=K H P^{-1}$, where $K$ is a positivedefinite symmetric matrix and satisfies the inequality

$$
H^{T}\left(A_{0} K+K A_{0}^{T}+N N^{T}+K M^{T} M K\right) H<0 .
$$

Thus, the matrices $H$ and $\bar{H}$ can be obtained by solving the above LMI. The result can be concluded by the following theorem.

Theorem 2. The sliding mode of the sliding mode control system (11) with the uncertain switching surface (7) is stable, if there exist a matrix $H$ of the orthogonal complement of $B_{0}$ and a positive-define matrix K such that the LMI (17) holds.

Remark 3. This result is similar to Theorem 1 proposed in [9]. Unlike the previous result, Theorem 2 shows a fact that the stability of the sliding mode is independent of the sliding surface design. It also means that its stability is invariant to the uncertainties of the sliding surface. In this way, the dynamics of the sliding mode can be designed separately for the SMC system (13).

Remark 4. It seems that there is no explicit sliding surface while deducing the sliding mode. Actually, the sliding surface cannot be arbitrarily selected. Its parameter should satisfy the constraint condition of $\bar{H}$, that is, $\Xi_{0} \bar{H}=O_{n \times(n-1)}$. Theorem 2 shows how to obtain $\bar{H}$ so that the normal sliding surface can be obtained. It is certainly to design the sliding surface first, since the solution of LMI (17) is not unique.

Then, the robust design issue is concentrated on the reaching phase design of the SMC system (14). In other words, the design objective (P1) is obtained, and the following design objective is to find a robust feedback control $u$ with the consideration of the uncertainties of the system and the sliding surface so that the new state variable $\sigma$ can converge to zero in finite time.

3.2. Reaching Phase Design. The dynamical motion of the reaching phase can be described by the differential equation of the state variable $\sigma$; that is,

$$
\begin{aligned}
\dot{\sigma}= & \Xi\left(A_{0}+N \widetilde{A} M\right) \bar{H} z_{1}+\Xi(A+N \widetilde{A} M) B_{0}\left(\Xi B_{0}\right)^{-1} \sigma \\
& +\Xi_{0} B_{0}(1+\widetilde{B}) u .
\end{aligned}
$$

With the definition of $\Xi$ and $\Gamma$, the differential equation (18) can be transformed into

$$
\begin{aligned}
\dot{\sigma}= & E_{n}^{T}\left(I_{n}+\widetilde{\Xi}\right) \Xi_{0}\left(A_{0}+N \widetilde{A} M\right) X \\
& +E_{n}^{T}\left(I_{n}+\widetilde{\Xi}\right) \Xi_{0} B_{0}(1+\widetilde{B}) u .
\end{aligned}
$$

To ensure every trajectory of the closed loop reaching the sliding surface in finite time, the reaching law should guarantee the reachability condition that can be checked by the sign of the time derivative of one positive definite function related to the sliding surface. The following positive definite function is used to check the finite time convergence about the sliding surface $S$ :

$$
V=\frac{1}{2} \sigma^{2}
$$

Its time derivative along $\sigma$ can be calculated as

$$
\begin{aligned}
\dot{V}= & E_{n}^{T} \Xi_{0} A_{0} X \sigma+E_{n}^{T}\left(\widetilde{\Xi} \Xi_{0} A_{0}+\Xi_{0} N \widetilde{A} M+\widetilde{\Xi} \Xi_{0} N \widetilde{A} M\right) X \sigma \\
& +E_{n}^{T} \Xi_{0} B_{0} u \sigma+E_{n}^{T}\left(\Xi_{0} B_{0} \widetilde{B}+\widetilde{\Xi} \Xi_{0} B_{0}+\widetilde{\Xi} \Xi_{0} B_{0} \widetilde{B}\right) u \sigma \\
\leq & E_{n}^{T} \Xi_{0} A_{0} X \sigma+\left\|E_{n}\right\| \\
& \times\left(\rho\left\|\Xi_{0} A_{0} X\right\|+(1+\rho)\left\|\Xi_{0} N\right\| \cdot\|M X\|\right)|\sigma| \\
& +E_{n}^{T} \Xi_{0} B_{0} u \sigma+q\left\|E_{n}\right\| \cdot\left\|\Xi_{0} B_{0}\right\| \cdot|u \sigma| .
\end{aligned}
$$




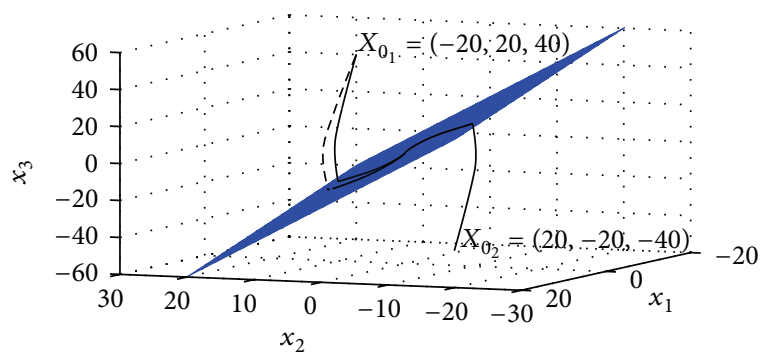

FIGURE 2: Trajectories with the normal sliding surface of $\varsigma=0$.

Suppose the reaching law to be

$$
\begin{aligned}
u=- & \frac{B_{0}^{T} \Xi_{0}^{T} E_{n}}{\left\|E_{n}\right\|^{2}\left\|\Xi_{0} B_{0}\right\|^{2}} \\
& \times\left\{E_{n}^{T} \Xi_{0} A_{0} X+\frac{1}{1-q}\right. \\
& \times\left[\kappa+(1+\rho)\left\|E_{n}\right\| \cdot\left\|\Xi_{0} N\right\| \cdot\|M X\|\right. \\
& \left.\left.+\rho\left\|E_{n}\right\| \cdot\left\|\Xi_{0} A_{0} X\right\|+\left\|E_{n}^{T} \Xi_{0} A_{0} X\right\|\right] \operatorname{sgn}(\sigma)\right\},
\end{aligned}
$$

where $\kappa$ is a positive number. Therefore, (21) can be rewritten as

$$
\begin{aligned}
\dot{V} \leq & -\frac{1}{1-q}\left(\kappa+\left\|E_{n}^{T} \Xi_{0} A_{0} X\right\|\right)|\sigma| \\
- & \frac{q}{1-q}\left[(1+\rho)\left\|E_{n}\right\| \cdot\left\|\Xi_{0} N\right\| \cdot\|M X\|\right. \\
& \left.\quad+\rho\left\|E_{n}\right\| \cdot\left\|\Xi_{0} A_{0} X\right\|\right]|\sigma| \\
& +\frac{q}{1-q}\left[\kappa+(1+\rho)\left\|E_{n}\right\| \cdot\left\|\Xi_{0} N\right\| \cdot\|M X\|\right. \\
& \left.+\rho\left\|E_{n}\right\| \cdot\left\|\Xi_{0} A_{0} X\right\|+\left\|E_{n}^{T} \Xi_{0} A_{0} X\right\|\right]|\sigma| \\
& +q\left\|E_{n}^{T} \Xi_{0} A_{0} X\right\| \cdot|\sigma| \\
\leq & -\kappa|\sigma|-(1-q)\left\|E_{n}^{T} \Xi_{0} A_{0} X\right\| \cdot|\sigma| .
\end{aligned}
$$

According to the Lyapunov method on finite-time convergence [5], $\sigma$ will reach zero in finite time with the reaching law (22). It means that the state of the system (14) will arrive at the sliding surface $S$ in finite time and remain on it. Thus, the design target (P2) is achieved. The result can be summarized in Theorem 5.

Theorem 5. For the uncertain system (14), any trajectory will approach the uncertain sliding surface (7) in finite time under the reaching law (22).

Theorem 5 shows that the sliding mode controller for the system (14) is obtained. Due to the invertibility of the invariant transformation (12), the sliding mode controller is also for

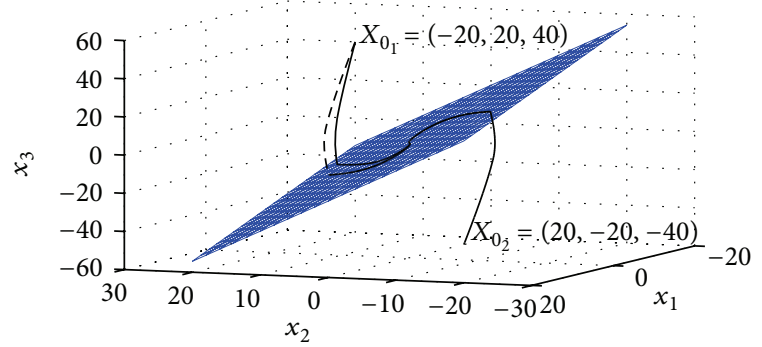

FIGURE 3: Trajectories with the sliding surface of $\varsigma=0.4$.

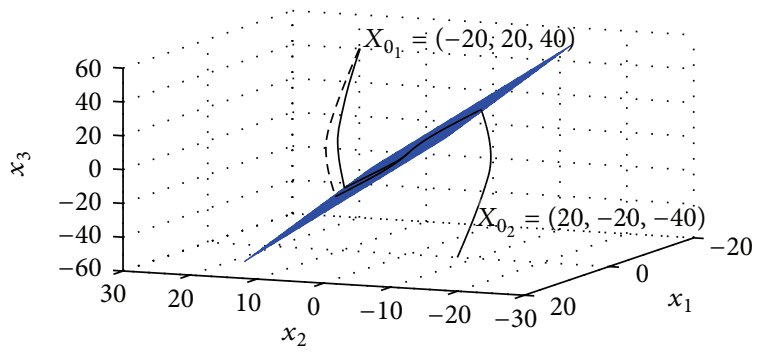

FIgURE 4: Trajectories with the sliding surface of $\varsigma=-0.4$.

the original uncertain system (6). Therefore, the sliding mode design with uncertain sliding surface is achieved.

\section{Numerical Example}

Consider the following uncertain SISO system:

$$
\begin{aligned}
& \dot{x}_{1}=(2+\cos t) x_{2}, \\
& \dot{x}_{2}=-x_{2}+x_{3}, \\
& \dot{x}_{3}=(1+\mu) u,
\end{aligned}
$$

where $\mu$ is the uncertain variable of the input vector with the range $[-0.2,0.2]$. The system (24) can be cast into the form of (11) with

$$
\begin{gathered}
N=\left[\begin{array}{ccc}
1 & 0 & 0
\end{array}\right]^{T}, \quad M=\left[\begin{array}{lll}
0 & 1 & 0
\end{array}\right], \\
\widetilde{A}=\cos t, \quad \widetilde{B}=\mu .
\end{gathered}
$$

Let the normal sliding surface be defined as

$$
S_{0} \triangleq\left\{X: \sigma_{0}(X)=x_{1}+2 x_{2}+x_{3}=0\right\} .
$$

Assume that the uncertainties occur in three terms during realization of the sliding surface. It can be rewritten as

$$
S \triangleq\left\{X: \sigma(X)=(1+\varsigma) x_{1}+(2+\varsigma) x_{2}+(1+\varsigma) x_{3}=0\right\},
$$




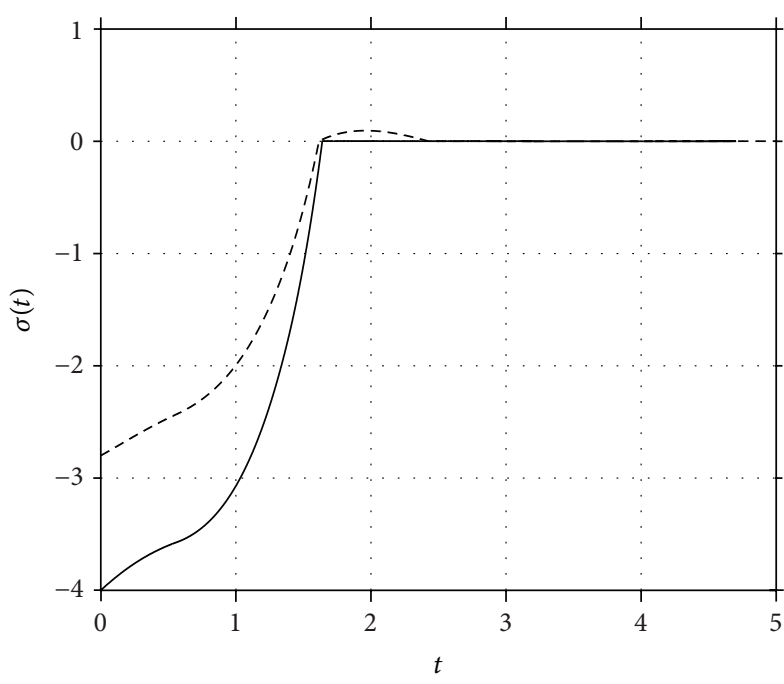

(a) Time responses of $\sigma(t)$ under the control (32)

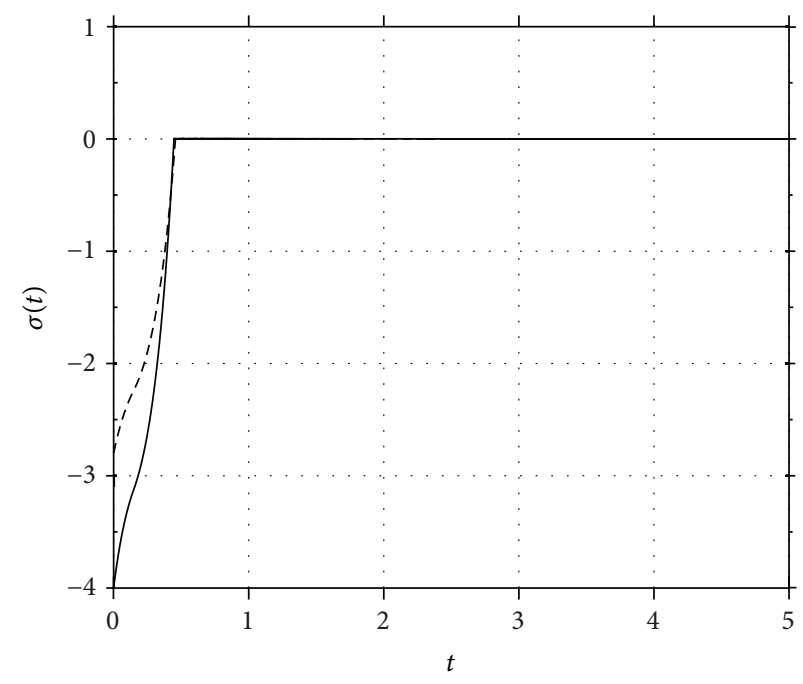

(b) Time responses of $\sigma(t)$ under the control (31)

FIGURE 5: Comparison with the time responses of the switching function.

where the uncertain variable $\varsigma$ varies from -0.4 to 0.4 . According to the assumption (A5), the structure of the sliding surface can be expressed as

$$
\Xi=\left[\begin{array}{lll}
1+\varsigma & 2+\varsigma & 1+\varsigma
\end{array}\right]=\frac{1}{3} E_{3}^{T}\left(I_{3}+\varsigma I_{3}\right)\left[\begin{array}{lll}
1 & 2 & 1 \\
1 & 2 & 1 \\
1 & 2 & 1
\end{array}\right] .
$$

Thus, $\epsilon=0.2, \rho=0.4$, and $q=0.68$.

Since the vectors $\left[\begin{array}{lll}1 & 0 & 0\end{array}\right]^{T}$ and $\left[\begin{array}{lll}0 & 1 & 0\end{array}\right]^{T}$ are all the basis of the null space of the vector $B_{0}=\left[\begin{array}{lll}0 & 0 & 1\end{array}\right]^{T}, H$ can be selected as

$$
H^{T}=\left[\begin{array}{lll}
1 & 0 & 0 \\
0 & 1 & 0
\end{array}\right]
$$

It can be checked that the sliding mode is stable with the following $\bar{H}$ :

$$
\bar{H}=\left[\begin{array}{lll}
1 & 0 & -1 \\
0 & 1 & -2
\end{array}\right]^{T} .
$$

Let $\kappa=0.1$. Then the sliding mode controller is

$$
u=-2 x_{3}-\frac{1}{0.32}\left(0.1+1.4\left|x_{2}\right|+2.8\left|x_{3}\right|\right) \operatorname{sgn}(\sigma) .
$$

The simulations are carried out from two start points: $X_{0_{1}}=(-20,20,40)$ and $X_{0_{2}}=(20,-20,-40)$. The results are shown in the following figures. The solid lines in all figures are of the case $\mu=1.2$. The dashed lines in all figures show the trajectories as $\mu=0.8$. Figure 2 shows the trajectories under the sliding mode controller (31) with the normal sliding surface. Figures 3 and 4 show the situations of $\varsigma=0.4$ and $\varsigma=-0.4$, respectively. All the trajectories reach the sliding surface under the reaching law, though the sliding surface varies. Moreover, the sliding modes in the simulations are all stable. Therefore, the sliding mode controller (31) can handle not only the uncertainties of the plant (24) but also the uncertainties of the sliding surface (27).

In order to show the advantage of the new robust design, the comparison in Figure 5 is carried out. Figure 5(a) shows the time responses of the switching function under the following sliding mode control designed without the consideration of the uncertainties of the sliding surface [2]:

$$
u_{0}=-\frac{1}{0.8}\left(0.1+\left|x_{2}\right|+2\left|x_{3}\right|\right) \operatorname{sgn}(\sigma) \text {. }
$$

The solid line is the result of the sliding mode controller $u_{0}$ with the normal sliding surface, while the dashed line illustrates the case in which the uncertainties of $\varsigma=-0.4$ occur for the switching function $\sigma(X)$. All trajectories start from the point $(-1,-1,-1)$ in the state space. The solid line approaches zero and remains on it. It means that the trajectories will remain on the sliding surface, once they reach it. However, it may not be true for the case that the uncertainties occur for the sliding surface, in which the trajectories cross the sliding surface. It implies the failure of the reaching phase design due to the uncertainties of the sliding surface. Under the same simulation conditions, Figure 5(b) shows the results of the robust sliding mode design (31). The dashed line and the solid line illustrate the normal case and the uncertain case with $\varsigma=-0.4$, respectively. It can be observed that the new reaching phase design is robust to the uncertainties of the sliding surface, since the trajectories remain on the sliding surface in the two simulation cases.

The simulation results verify the results of this paper. Moreover, they prove the fact again that one reaching law of sliding mode controller may correspond to several sliding surfaces and that the robustness of the reaching law can be used to overcome the uncertainties of the sliding surface. 


\section{Conclusions}

A new design methodology of SMC is proposed to handle the important design situation that there exist uncertainties in the sliding surface. The invariant transformation is used to separate the sliding mode and the reaching mode of the SMC design such that it is not necessary to consider the uncertainties of the sliding surface during the sliding phase design. The new controller explores the robustness of the reaching law design so that the proposed sliding mode controller can handle not only the uncertainties of the plant but also the uncertainties of the sliding surface. Obviously, the results of this paper can be extended for more general systems and to design the sliding mode controller with several uncertain sliding surfaces.

\section{Acknowledgments}

This work was supported by the Chinese National Science Foundation (no. 60736022), the Doctoral Fund of Ministry of Education of China (no. 20102125120001), and the Fundamental Research Funds for the Central Universities of China (no. 3132013331).

\section{References}

[1] V. I. Utkin, "Variable structure systems with sliding modes," IEEE Transactions on Automatic Control, vol. 22, no. 2, pp. 212222, 1977.

[2] V. I. Utkin, Sliding Modes in Control and Optimization, Communications and Control Engineering Series, Springer, Berlin, Germany, 1992.

[3] A. S. I. Zinober, Variable Structure and Lyapunov Control, vol. 193 of Lecture Notes in Control and Information Sciences, Springer, London, UK, 1994.

[4] K. D. Young, V. I. Utkin, and Ü. Özgüner, "A control engineer's guide to sliding mode control," IEEE Transactions on Control Systems Technology, vol. 7, no. 3, pp. 328-342, 1999.

[5] Y. V. Orlov, Discontinuous Systems-Lyapunov Analysis and Robust Synthesis under Uncertainty Conditions, Communications and Control Engineering Series, Springer, London, UK, 2009.

[6] S. Yu, X. Yu, B. Shirinzadeh, and Z. Man, "Continuous finitetime control for robotic manipulators with terminal sliding mode," Automatica, vol. 41, no. 11, pp. 1957-1964, 2005.

[7] S.-C. Tan, Y. M. Lai, and C. Tse, "General design issues of sliding-mode controllers in DC-DC converters," IEEE Transactions on Industrial Electronics, vol. 55, no. 3, pp. 1160-1174, 2008.

[8] W.-C. Su, S. V. Drakunov, and Ü. Özgüner, "Constructing discontinuity surfaces for variable structure systems: a Lyapunov approach," Automatica, vol. 32, no. 6, pp. 925-928, 1996.

[9] H. H. Choi, "An explicit formula of linear sliding surfaces for a class of uncertain dynamic systems with mismatched uncertainties," Automatica, vol. 34, no. 8, pp. 1015-1020, 1998.

[10] C.-C. Wen and C.-C. Cheng, "Design of sliding surface for mismatched uncertain systems to achieve asymptotical stability," Journal of the Franklin Institute, vol. 345, no. 8, pp. 926-941, 2008.
[11] V. Acary, B. Brogliato, and Y. V. Orlov, "Chattering-free digital sliding-mode control with state observer and disturbance rejection," IEEE Transactions on Automatic Control, vol. 57, no. 5, pp. 1087-1101, 2012.

[12] Y. Yao, K. Zheng, K. Ma, and T. Shen, "Sliding mode control design with a class of piecewise smooth switching surfaces," International Journal of Modelling, Identification and Control, vol. 7, no. 3, pp. 294-304, 2009.

[13] K. Zheng, T. Shen, and Y. Yao, "New approaching condition for sliding mode control design with Lipschitz switching surface," Science in China. Series F, vol. 52, no. 11, pp. 2032-2044, 2009.

[14] A. F. Filippov, "Differential equations with discontinuous righthand side," American Math Society Translation, no. 2, pp. 354362, 1964.

[15] D. Giaouris, S. Banerjee, B. Zahawi, and V. Pickert, "Stability analysis of the continuous-conduction-mode buck converter via Filippov's method," IEEE Transactions on Circuits and Systems I, vol. 55, no. 4, pp. 1084-1096, 2008.

[16] P. P. Khargonekar, I. R. Petersen, and K. Zhou, "Robust stabilization of uncertain linear systems: quadratic stabilizability and $H_{\infty}$ control theory," IEEE Transactions on Automatic Control, vol. 35, no. 3, pp. 356-361, 1990. 


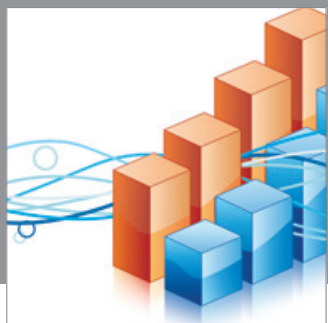

Advances in

Operations Research

mansans

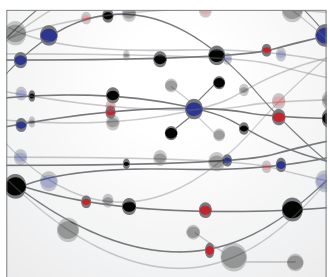

The Scientific World Journal
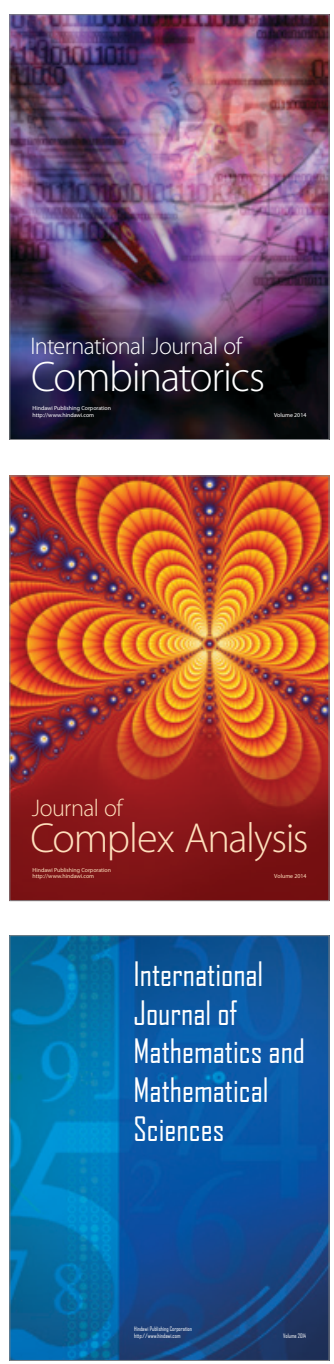
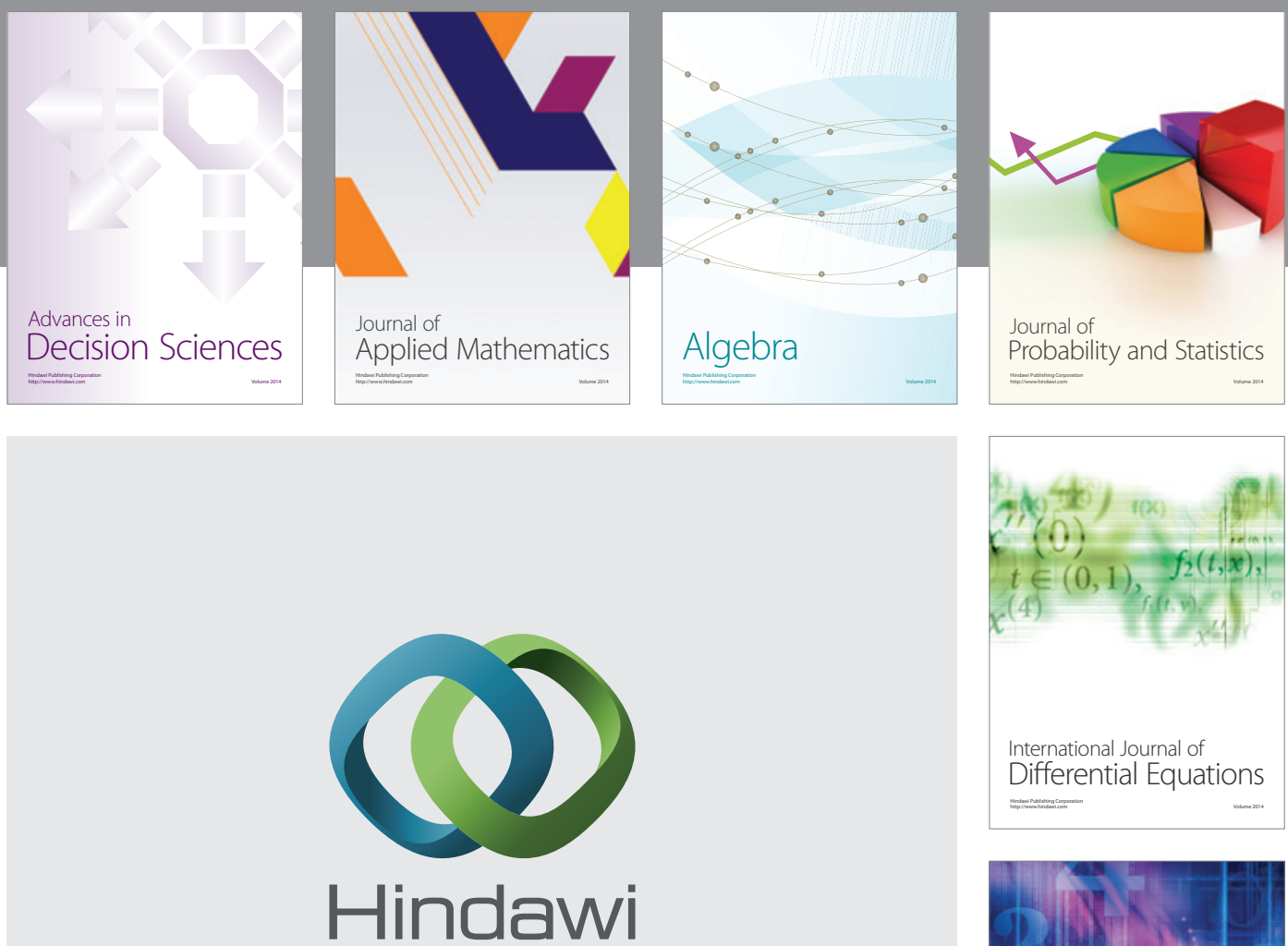

Submit your manuscripts at http://www.hindawi.com
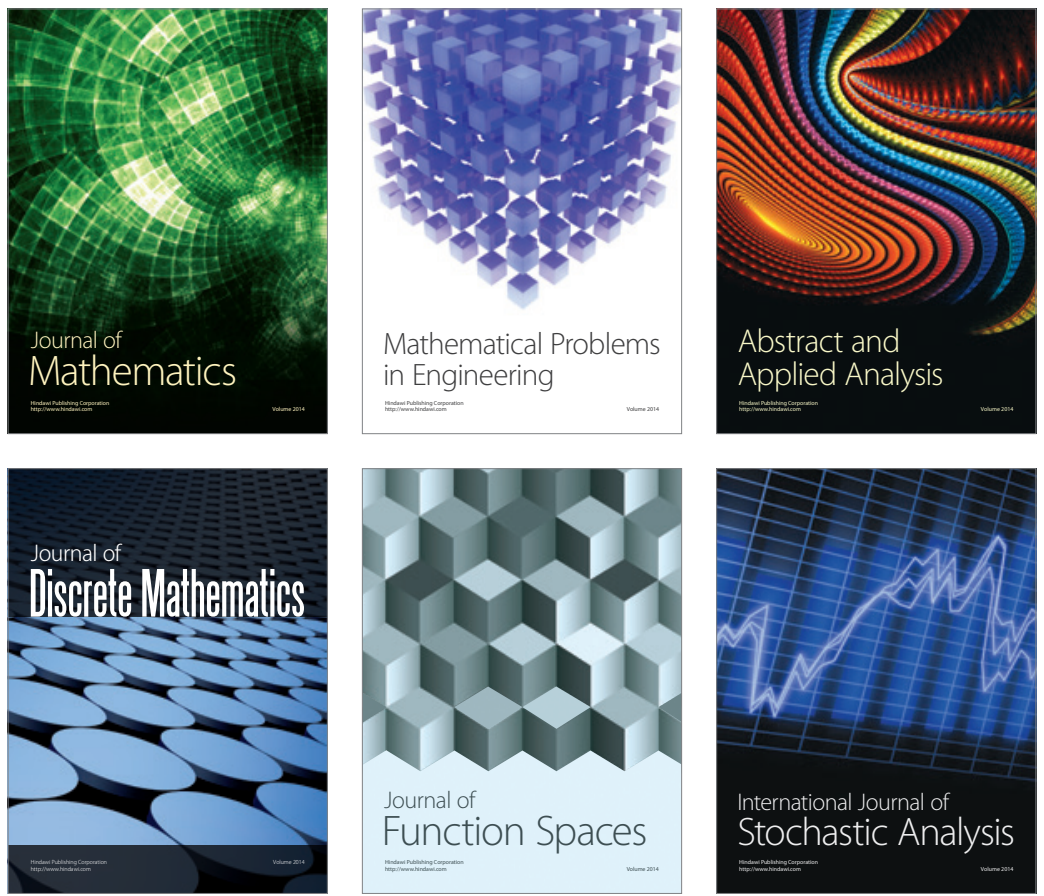

Journal of

Function Spaces

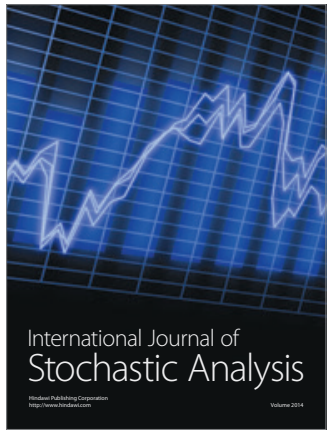

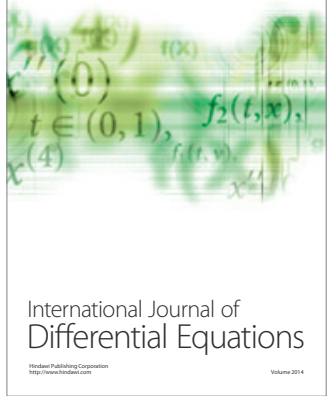
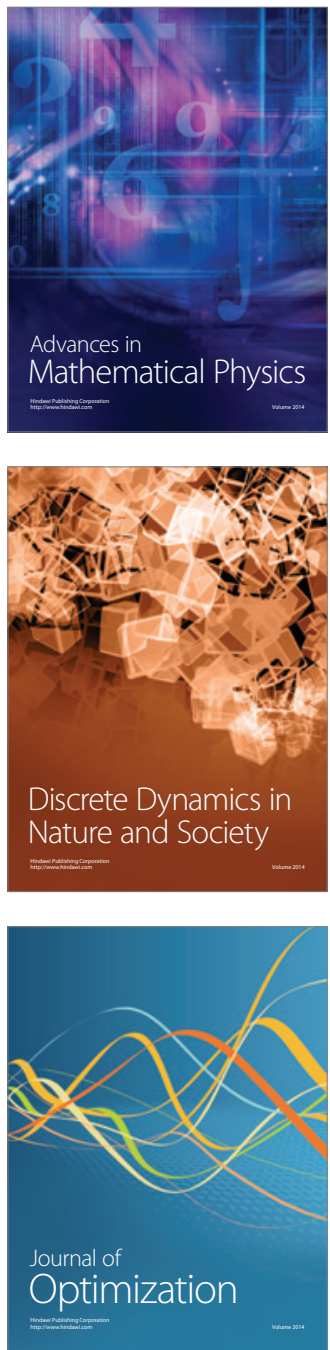